Eng.Amin ESHGHI,MSc

E-mail: a.eshghi9@ hotmail.com

Department of Industrial Engineering

Faculty of Engineering, Shahed University, Tehran, Iran

Associate Professor Seyed Meysam MOUSAVI, PhD

E-mail: sm.mousavi@shahed.ac.ir (*Corresponding author)

Department of Industrial Engineering

Faculty of Engineering, Shahed University, Tehran, Iran

Professor Jurgita ANTUCHEVICIENE, PhD

E-mail: jurgita.antucheviciene@vgtu.It

Department of Construction Management and Real Estate, Faculty of

Civil Engineering, Vilnius Gediminas Technical University, Lithuania

Vahid MOHAGHEGHI, PhD Student

E-mail: v.mohagheghi@ hotmail.co

Faculty of Engineering, Shahed University, Tehran, Iran

\title{
EARNED VALUE ANALYSIS APPROACH BY EXTENDING NEW KEY PERFORMANCE INDICATORS IN A NEW FRAMEWORK UNDER A GREY ENVIRONMENT
}

Abstract. An inseparable part of project management is the existence of uncertainty. There are various methodologies and tools to handle the impact of uncertainty on project performance.Todenote the uncertainty, we use grey theory because ofits accuracy and convenience in modeling uncertainty. In this paper, a new grey-based earned value technique with a new extended set of key performance indicators (KPIs)is proposed to measure the progress, performance, cost and time estimate at completion of the construction projects under uncertainty circumstances. A new formulation of cost and time estimation at completion is introduced that is based on using a newly developed weighting method called WTODIM. The proposed model improves managing different aspects of a project through a flexible and intelligent approach. Finally, a case study of construction project from the literature is presented to show the efficiency of presented model.

Keywords:Uncertainty, Earned value management, Key performance indicators(KPIs), Grey theory, Construction projects

JEL Classification: C02, C44, D81, L74, M10. 
Amin Eshghi, Seyed M. Mousavi, Jurgita Antucheviciene, Vahid Mohagheghi

\section{Introduction}

Always, construction projectsare faced with many risks to achieve predetermined objectives over their life cycle.Dynamism, complexities and specific nature of construction projects are quickly deteriorated andrisks could behazardousfor project components if not properly reduced or removed(Taillandieret al., 2015; Rostami et al., 2015).Risks come from uncertainties that exist in all projects.Project risk definition could be a vague status or an event which has a positive or negative effect on goals of a project (PMI, 2013).Since uncertainty is one of the risk factors, the term uncertainty should be described and explained.

Due to increasing costs and complexity in the projects, as well as growing uncertainty and existing risks, project managers employ risk management techniques in the planning and control of projects to reduce the risk and diversion of the project from the predesignated goals. The range of uncertainty in projects is significant and many of the project management activities, focus on defining and deciding on a set of possible measures against project's uncertainties from the early stages of the project life cycle. Part of the uncertainty in projects, refer topossibility to change the project performance criteria, such as cost, time and quality.Uncertainties can also be found on issues, such as ambiguity and unpredictability in understanding project teamwork and stakeholder's behavior, lack of information, lack of an explicit structure to consider project issues,known and unknown resources of deviations in the project and other related issues(Banihashemi et al., 2017; Anantatmula and Fan, 2018).

Two types of uncertainties can be categorized inreal-world problems that are random uncertainty and perceptual uncertainty. To describe and study the aspect of the second type, grey theory has been developed in situations with low data or incomplete qualitative information. Grey theory works well in fuzzy conditions (Deng, 1989). Grey systems have been named based on thecolor of subjects under investigation. So that the colors brightness rate represents the clarity level of information and data. Accordingly, systems with well-defined information are white systems, systems with ill-defined data are black systems and systems with somewhat known and partly unknown information are called grey systems ( $\mathrm{Li}$ and Liu, 2008; Li et al., 2014).

Lin et al.(2004) looked at the state-of-the-art of the grey system theory and its applications since it's creation. Then, its several successful applications were mentioned by examining the history of this theory.Grey mathematical 


\section{Earned Value Analysis Approach by Extending New Key Performance Indicators in a New Framework under a Grey Environment}

programming models were employed for project time-cost-quality trade-offs with uncertain conditions by RazaviHajiaghaet al. (2015).Rahimnia et al.(2011) applied a methodology to evaluate the qualitative quiddity of organizational attitudes. As an aim of their study, vision of several universities was assessed and ranked by the grey theory.Slavek and Jović(2012) employed grey theory to anticipate and specify software projects ranking by their success and then evaluated the quality of them.Bai and Sarkis(2013) presented a method for evaluating critical success factors (CSFs) for implementing business process management (BPM), using a new gray-based decision-making approach. It can efficiency use uncertainty and indistinctive problems in order to help project managers making appropriate investment decisions for the BPM.Bhattacharyya (2015) presented a model based on multiple attributes decision making(MADM) methodology to select project portfolio for research and development(R\&D). Firstly, the study discussed the uncertainty which exists on the preferences of decision-makers about alternatives or attributes of the project. Secondly as a coping with the uncertainty, the grey theory is applied to the project ranking.Prioritizing entrepreneurship main risks in non-profit financial funds based on TODIM method under grey environment in Iran was carried out by Ekhtiari et al. (2016).

Monitoring of project performance information has become an extremely critical taskdue to complexity of the activities and conditions affecting the project. In this paper, key performance indicators (KPIs) are employed to evaluate the power of project success in EVM.EVMassists the project manager to handleproject from two perspectives. The first one is to acknowledgeexisting performance indicators, and the second one is to present future prediction (Noori et al., 2008).Thus, by reviewing the research, it is noticed that in the project control and management, the indicators and estimates, weights of the indices from the MADMperspectivesand the uncertainty with consideration of the grey theory in the analysis of the earned value management (EVM) are not considered. Therefore, in this paper, taking into account the assumptions mentioned, a new attempt is made to analyze the earned value, which is closer to real life conditions of construction projects.

In summary, the main features ofthis paper that separate it from the similar studies in thisfield are as follows:

- Grey theory is presented and applied inproject performance monitoring and analyzing. It provides the decision maker (DM) with more flexibility in expressing uncertainty. Furthermore, the project performance calculations would bemore flexible, simple, and convenience. 
Amin Eshghi, Seyed M. Mousavi, Jurgita Antucheviciene, Vahid Mohagheghi

- A new set of KPIs is presented to handle and evaluate a construction project.Moreover,these KPIs are separated into two groups of project implementation and risk indicators.For this purpose, five KPIs of critical aspectsare coupled with additional four KPIs of risk.It should be mentioned that KPIs of risk constitute different risk indicators for cost and time.

- Weight of each KPI in grey-based estimation equations is computed by developing new concept of iterative multi-criteria analysis method called TODIM (an acronym in Portuguese for interative multi-criteria decision making). In other words, the importance of KPIs is both denoted by direct judgement of the DMs and by computing weights based on the gatheredjudgements.

To conceive comprehension, the rest of this paper is divided into 4 sections. A brief overview on grey numbers and grey-based EVM method are given in section 2 . The proposed analytical approach is introduced in section 3 . Then, a case study from the literature is presented and solved in section 4. Discussion of result is represented in section5. Finally, the paper is concluded in the last section.

\section{Presented greyEVM calculations}

The EVM is a powerful tool that permits practitioners, project managers, and others to monitorthe project condition during the life cycle of project. It is possible to gain more efficiency in managing the handle of project, programs, and portfolios. The opportunities are measuredobviously by EVM to keep monitoring over important issues, such as budget, scope, and schedule of different types of projects (Salari etal., 2014; Simion and Marin, 2018).

Measuring EV is not simple and how to determine it is a matter of discussion between EVM practitioners. The basic concept of EVM is to compare the amount of earned value against the money paid for each activity. Firstly, each activity progress is determined by expert opinion and they have to be transformed into the grey progress percent by means of linguistic terms showed in Table1.

$\otimes G P P_{i}=\left[\underline{a_{i}} \cdot \overline{a_{i}}\right]$

Secondly, a formal definition of grey-based earned value for activity $i$ can be obtaining as follows:

$\otimes E V_{i}=\otimes G P P_{i} * B A C_{i}=\left[\underline{E_{i}} \cdot \overline{E_{i}}\right]=\left[a_{i} * B A C_{i} \cdot a_{i} * B A C_{i}\right]$ 


\section{Earned Value Analysis Approach by Extending New Key Performance Indicators in a New Framework under a Grey Environment}

where $i=1,2, \ldots, n$ is the total number of project activities and $\mathrm{BAC}_{\mathrm{i}}$ denots the Budget of Completion.

The overall grey-based earned value could be calculated by rolling up all the $\otimes E V_{i}$ for activity as follows:

$\otimes E V_{O}=\sum_{i=1}^{n} \otimes E V_{i}=\left[\sum_{i=1}^{n} \underline{E_{i}} \cdot \sum_{i=1}^{n} \overline{E_{i}}\right]=\left[\underline{E_{1}} \cdot \overline{E_{2}}\right]$

Table 1. Dedicated linguistic terms associated with grey numbers

\begin{tabular}{ll}
\hline Linguistic terms & Grey numbers \\
\hline Near to the beginning & {$[0,0.1]$} \\
Very low & {$[0.1,0.2]$} \\
Low & {$[0.2,0.3]$} \\
Less than half & {$[0.3,0.4]$} \\
Half & {$[0.4,0.5]$} \\
More than half & {$[0.5,0.6]$} \\
High & {$[0.6,0.7]$} \\
Very high & {$[0.7,0.8]$} \\
Near to the end & {$[0.8,1]$} \\
\hline
\end{tabular}

Subsequently, according to PMI (2013), cost performance index(CPI), schedule performance index (SPI), schedule cost index (SCI) and cost etimation at completion (EAC) grey-based calculations are presented using Eqs.(4)-(8):

$$
\begin{aligned}
& \otimes S P I=\otimes E V_{O} /_{P V}=\left[E_{1} / P V \cdot \overline{E_{2} / P V}\right] \\
& \otimes C P I=\otimes E V_{O} /_{A C}=\left[E_{1} /_{A C} \cdot \overline{E_{2} / A C}\right] \\
& \otimes S C I=\otimes C P I \times \otimes S P I \\
& =\left[\begin{array}{l}
\min (\underline{C P I} \times \underline{S P I} \cdot \underline{C P I} \times \overline{S P I} \cdot \overline{C P I} \times \underline{S P I} \cdot \overline{C P I} \times \overline{S P I}) . \\
\max (\underline{C P I} \times \underline{S P I} \cdot \underline{C P I} \times \overline{S P I} \cdot \overline{C P I} \times \underline{S P I} \cdot \overline{C P I} \times \overline{S P I})
\end{array}\right]
\end{aligned}
$$

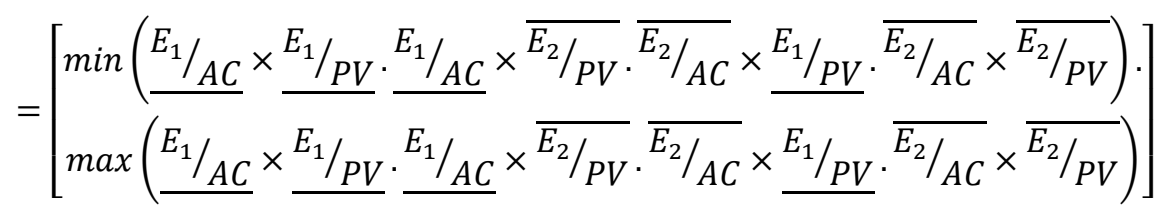

$$
\begin{aligned}
& =[\underline{S C} \cdot \overline{S C}]
\end{aligned}
$$


Amin Eshghi, Seyed M. Mousavi, Jurgita Antucheviciene, Vahid Mohagheghi

$$
\begin{aligned}
& \otimes E A C=\frac{B A C}{\otimes C P I}=[B A C \cdot B A C] \times\left[\frac{1}{\underline{C P I}} \cdot \frac{1}{\overline{C P I}}\right]=[\underline{e a c} \cdot \overline{e a c}] \\
& \otimes E A C=A C+\left[\frac{B A C-\otimes E V_{O}}{\otimes S C I}\right] \\
& =A C+\left[\min \left(\begin{array}{l}
\left(B A C-E_{2}\right) \times \frac{1}{\frac{S C}{1}} \cdot\left(B A C-E_{2}\right) \times \frac{1}{\overline{S C}} \cdot \\
\left(B A C-E_{1}\right) \times \frac{1}{\underline{S C}} \cdot\left(B A C-E_{1}\right) \times \frac{1}{\overline{S C}}
\end{array}\right) .\right. \\
& \left.\max \left(\begin{array}{l}
\left(B A C-E_{2}\right) \times \frac{1}{\frac{S C}{1}} \cdot\left(B A C-E_{2}\right) \times \frac{1}{\overline{S C}} \cdot \\
\left(B A C-E_{1}\right) \times \frac{1}{\underline{S C}} \cdot\left(B A C-E_{1}\right) \times \frac{1}{\overline{S C}}
\end{array}\right)\right]
\end{aligned}
$$

where PV and AC are planned value and actual cost, respectively.

Due to lack of attention to time units in EVM calculations, three methods have been introduced to evaluate the schedule performance. Lipke(2003) proposed the earned schedule (ES)totranslate the EV of a given condition date interms of time units. In other words, principles for ES are exactly similar to earned value. New formula forthe ES under the grey environment is determined as below:

$E S=t+\left[\frac{E V_{i}-P V_{t}}{P V_{t+1}-P V_{t}}\right] \mathrm{i}=1,2$

$\otimes E S=\left[E S_{1} \cdot E S_{2}\right]$

where $t$ is denoted as a value so that $P V_{t}<E V<P V_{t+1}$.In other words, $t$ represents the closest period which the current $\mathrm{EV}$ is more than the planned value of that period.

With ES, a different index for project schedule performance can be calculated which is called $S P I_{(t)}$ :

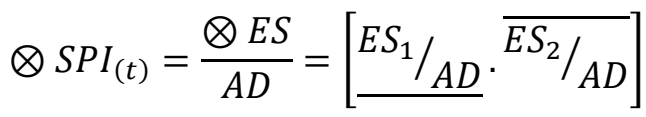

General formula for time estimation at completion is represented by Eq. (11) which performance factor $(\mathrm{PF})$ denoted by completion trend of remained activities

$\otimes E A C_{(t)}=A D+\left[\frac{P D-\otimes E S}{P F}\right]$

- $\mathrm{PF}=1$ : The duration of the remained activities is as planned.

$\otimes E A C_{(t)}=A D+[P D-\otimes E S]=\left[A D+P D-\otimes \overline{e s} . A D+P D-E S_{1}\right]$ 
- $P F=\otimes S P I_{(t)}:$ The trend of $\otimes S P I_{(t)}$ changes the activities duration which did not completed.

$$
\begin{aligned}
& \otimes E A C_{(t)}= A D+\left[\frac{P D-\otimes E S}{\otimes S P I_{(t)}}\right] \\
& \min \left(\begin{array}{l}
\left(P D-E S_{2}\right) \times \frac{1}{\frac{S P I_{(t)}}{1}} \cdot\left(P D-E S_{2}\right) \times \frac{1}{\overline{S P I_{(}(t)}} \cdot \\
\left.\left(P D-E S_{1}\right) \times \frac{1}{\frac{S P I_{(t)}}{1}} \cdot\left(P D-E S_{1}\right) \times \frac{1}{\overline{S P I_{(t)}}}\right)
\end{array}\right) \\
& \max \left(\begin{array}{l}
\left(P D-E S_{2}\right) \times \frac{1}{\frac{S P I_{(t)}}{1}} \cdot\left(P D-E S_{2}\right) \times \frac{1}{\overline{S P I_{(t)}}} \cdot \\
\left.\left(P D-E S_{1}\right) \times \frac{1}{S P I_{(t)}} \cdot\left(P D-E S_{1}\right) \times \frac{1}{\overline{S P I_{(t)}}}\right)
\end{array}\right]
\end{aligned}
$$

- $\mathrm{PF}=\otimes S C I_{(t)}$ : The trend of $\otimes S C I_{(t)}$ changes the activities duration which didnot complete.

$$
\begin{aligned}
& \otimes S C I_{(t)}=\otimes S P I_{(t)} \times \otimes C P I \\
& =\left[\min \left(\underline{S P I_{(t)}} \times \underline{C P I} \cdot \underline{S P I_{(t)}} \times \overline{C P I} \cdot \overline{S P I_{(t)}} \times \underline{C P I} \cdot \overline{S P I_{(t)}} \times \overline{C P I}\right) .\right. \\
& \left.\max \left(\underline{S P I_{(t)}} \times \underline{C P I} \cdot \underline{S P I_{(t)}} \times \overline{C P I} \cdot \overline{S P I_{(t)}} \times \underline{C P I} \cdot \overline{S P I_{(t)}} \times \overline{C P I}\right)\right]
\end{aligned}
$$

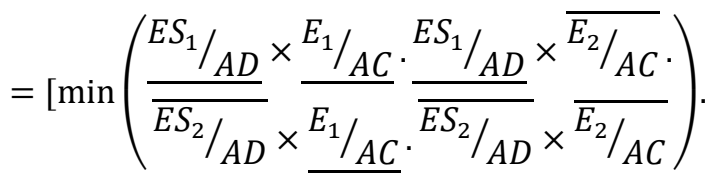

$$
\begin{aligned}
& \left.\max \left(\frac{E S_{1} /_{A D}}{\overline{E S_{2} / A D}} \times \frac{E_{1} /_{A C}}{E_{1} / A C} \cdot \frac{E S_{1} / A D}{\overline{E S_{2} / A D}} \times \frac{\overline{E_{2} / A C}}{E^{E_{2} / A C}}\right)\right]=\left[S C_{(t)} \cdot \overline{S C_{(t)}}\right]
\end{aligned}
$$

Regarding to $\otimes S C I_{(t)}$ as a performance factor,the $\otimes E A C_{(t)}$ is calculated as follows: 
Amin Eshghi, Seyed M. Mousavi, Jurgita Antucheviciene, Vahid Mohagheghi

$$
\begin{aligned}
& \otimes E A C_{(t)}=A D+\left[\frac{P D-\otimes E S}{\otimes S C I_{(t)}}\right]=A D+\left[\frac{\left(P D-E S_{2} \cdot P D-E S_{1}\right)}{\left(\frac{E S_{1}}{A D} \cdot \frac{E S_{2}}{A D}\right)}\right] \\
& =A D+\left(\begin{array}{l}
\min \left[\frac{P D-E S_{2}}{\frac{E S_{1}}{A D}} \cdot \frac{P D-E S_{2}}{\frac{E S_{2}}{A D}} \cdot \frac{P D-E S_{1}}{\left.\frac{E S_{1}}{A D} \cdot \frac{P D-E S_{2}}{\frac{E S_{2}}{A D}}\right] .}\right. \\
+\max \left[\frac{P D-E S_{2}}{\frac{E S_{1}}{A D}} \cdot \frac{P D-E S_{2}}{\frac{E S_{2}}{A D}} \cdot \frac{P D-E S_{1}}{\frac{E S_{1}}{A D}} \cdot \frac{P D-E S_{2}}{\frac{E S_{2}}{A D}}\right]
\end{array}\right)
\end{aligned}
$$

Where $\mathrm{AD}=$ Actual duration, $\mathrm{PD}=$ Planned duration

\section{Proposed methodology}

\subsection{KPIs overview and calculations}

As regards to the fact that the tools of this study for evaluating the project performance are based on KPIs, definition of KPIs is significant to provide a preliminary understandingof the implemented method.KPIsconcentrate on differentaspects of project performance, output,and outcomes, leading tosuccess of the project. Any project will only have KPIs in its area, if a KPI is significant to the project. Indeed, KPIs are project-based (Wiley, 2015). The process of choosing the appropriate KPIs would be difficult,whereas determining KPIs or creating an archive or set of KPIs is easy (Kerzner, 2017).

Sometimes, information and data collection methods must be extended at the same time as the project progresses, but in this sub-section, generally we introduce the following KPIs in construction projects:cost performance index (CPI), schedule performance index (SPI), quality performance index(QPI), stakeholder satisfaction performance indicator (SSPI), economical performance indicator (EPI), political performance indicator (PPI), management performance indicator (MPI), construction performance indicator (CsPI), cost risk performance indicator(CRPI), time risk performance indicator(TRPI).

Generally, significance of the indicators and their influential factors can be proved and disproved by the judgment of experts. The value of a particular KPI is determined by rolling up the normalized individual weight ofaninfluential factor(IF) multiplied by its value $\left(F_{i}\right)$; where IF values and IF weightings are obtained through linguistic tables and weighting methods, respectively. It should be noted that the values of grey RPIs $(\otimes C R P I$ or $\otimes T R P I)$ are calculated by adding four individual group values multiplied with their respective normalized group weightings. For instance, the $\otimes Q P I$ and $\otimes C R P I$ are obtained as follows: 


\section{Earned Value Analysis Approach by Extending New Key Performance Indicators in a New Framework under a Grey Environment}

$$
\begin{aligned}
& \otimes Q P I=\sum_{i=1}^{n}\left(\otimes \alpha_{Q_{i}} \times \otimes F_{i}\right) \\
& \otimes C R P I=\left(\otimes w_{1} \times \otimes E P I\right)+\left(\otimes w_{2} \times \otimes C o P I\right)+ \\
& \quad\left(\otimes w_{3} \times \otimes P P I\right)+\left(\otimes w_{4} \times \otimes M P I\right)+\left(\otimes w_{5} \times \otimes C S P I\right)
\end{aligned}
$$

Where $\otimes \alpha_{K P I_{i}}$ is internal weightings of the respective IFs, which determined by $\mathrm{DMs} ; \otimes F_{i}$ are values of IFs(ranging from $[0,0$.$\left.] to [0.9,1]\right) ; \otimes w_{i}(i=1, \ldots .5)$ and $\otimes z_{j}(j=1 . . .5)$ are weights of constituent KPIs in $\otimes C R P I$ and $\otimes T R P I$ calculated by means of relative importance index(RII). According to Muhwezi(2014), the RII formula is computed by followingEq.:

$\otimes R I I=\frac{\sum \otimes W}{A \times N}$

$\otimes W=$ Assigned weight forany factor (Table 1);

$\mathrm{A}=$ Highest weight (i.e. $[0.9,1]$ in this case) and;

$\mathrm{N}=$ Total number of respondents.

Table 2 shows the scale of attribute weights and ratings.

Table 2. The scale of attribute weights $\otimes w$ and attribute ratings $\otimes A$

\begin{tabular}{llll}
\hline Scale & $\otimes w$ & Scale & $\otimes A$ \\
\hline Very low (VL) & {$[0.0,0.1]$} & Very poor (VP) & {$[0,1]$} \\
Low (VL) & {$[0.1,0.3]$} & Poor (P) & {$[1,3]$} \\
Medium low (ML) & {$[0.3,0.4]$} & Medium poor (MP) & {$[3,4]$} \\
Medium (M) & {$[0.4,0.5]$} & Fair (F) & {$[4,5]$} \\
Medium high (MH) & {$[0.5,0.6]$} & Medium good (MG) & {$[5,6]$} \\
High (H) & {$[0.6,0.9]$} & Good (G) & {$[6,9]$} \\
Very high(VH) & {$[0.9,1]$} & & \\
\hline
\end{tabular}

\section{2. $\otimes \boldsymbol{G I E A C _ { ( \$ ) } \text { calculations }}$}

New grey improved cost estimate at completion $\left(\otimes G I E A C_{(\$)}\right)$ is presented as follows:

$\otimes \operatorname{GIEAC}_{(\$)}=A C+$

$B A C-\otimes E V_{O}$

$\overline{\left(\otimes W K_{1} \times \otimes C P I+\otimes W K_{2} \times \otimes S P I+\otimes W K_{3} \times \otimes Q P I+\otimes W K_{4} \times \otimes S F P I+\otimes W K_{5} \times \otimes C R P I\right)}$ where $\widetilde{W K}_{i}$ denotes the weighting of each KPI used in the formation of $\otimes$ GIEAC $(\$)$ and is obtained by W-TODIMmethod, which is proposed in the following: 
Amin Eshghi, Seyed M. Mousavi, Jurgita Antucheviciene, Vahid Mohagheghi

Steps of proposed W-TODIM method:In this paper, a method of TODIM for weighting (called W-TODIM) is introduced for assigning weight to each KPI according to the DM's opinion. The recognized method named presented by Gomes and Lima (1992) has been utilized to solving MADM problems based on the Prospect theory.

As the basic step of the W-TODIM method, consider on MADM problem with a set of six key performance indicators $K P I=$ $\{C P I . S P I . Q P I . S S P I . S F P I . R P I\}$ and a set of $m \mathrm{DMs}, D M=\left\{D M_{1} . D M_{2} \cdot \cdots\right.$ .$\left.D M_{m}\right\}$, whose $W=\left[W_{1} \cdot W_{2} \cdot \cdots \cdot W_{6}\right]^{T}$ is the weight vector of the KPIs such that $0 \leq W_{i} \leq 1$ for all $i=1 . \cdots .6$.

The procedure of W-TODIM method is presented in the following steps:

Step 1. Establish DM's opinion matrix for importance of each KPI. Noted that, the values are nominal at first. Numerical matrix is then performed using Table 1. Moreover, the evaluation of $K P I_{i}$ with respect to each DM $\left(D M_{j}\right)$ is denoted by $W_{i j}$, $i=1.2 \ldots .6, j=1.2 \ldots . m$. For convenience, the decision matrix is denoted by $W=\left(W_{i j}\right)_{6 \times m}$.

Step 2.The relative weight(subjective preference) $S P_{r j}$ for each $\mathrm{DM}, D M_{j}$, is calculated with respect to the reference DM (best DM in terms of science, background, and so on) $S P_{r}$ as $S P_{r j}=\frac{S P_{j}}{S P_{r}}, j=1.2 \ldots . m$, where $S P_{r}=\max _{j}\left\{S P_{j}\right\}$.

Step 3. For each key performance indicators pair $K P I_{u}$ and $K P I_{v}$, calculate the perceived dominance degree of $K P I_{u}$ over $K P I_{v}$ with respect to $D M_{j}$ by:

$Q_{u v}^{j}=Q_{j}\left(K P I_{u} \cdot K P I_{v}\right)=\left\{\begin{array}{cl}\sqrt{\frac{\left(y_{u j}-y_{v j}\right) S P_{r j}}{\sum_{j=1}^{m} S P_{r j}}} & \text { if } y_{u j}-y_{v j}>0 \\ 0 & \text { if } y_{u j}-y_{v j}=0 \\ -\frac{1}{\theta} \sqrt{\frac{\left(y_{u j}-y_{v j}\right) \sum_{j=1}^{m} S P_{r j}}{S P_{r j}}} & \text { if } y_{u j}-y_{v j}<0\end{array}\right.$

$u . v=1.2 \cdot \cdots \cdot n, j=1.2 \cdot \cdots \cdot m$,

Where $\theta>0$ is defined as the attenuation factor of the loss and a larger $\theta$ means a smaller degree of loss aversion. Moreover, $\left(y_{u j}-y_{v j}\right)>0$ denotes the gain of $K P I_{u}$ over $K P I_{v}$ with respect to $D M_{j}$, while $y_{u j}-y_{v j}<0$ denotes the loss of $K P I_{u}$ over $K P I_{v}$ with respect to $D M_{j} . u . v=1.2 \ldots . n, j=1.2 \ldots . m$.

Step 4.Calculate the collective perceived dominance degree for each alternative pair $K P I_{u}$ and $K P I_{v}$ by: 


\section{Earned Value Analysis Approach by Extending New Key Performance} Indicators in a New Framework under a Grey Environment

$$
\gamma_{u v}=\sum_{j=1}^{m} Q_{u v}^{j} . \quad u . v=1.2 \cdot \cdots \cdot m .
$$

Step 5. Compute the overall performance degree of $K P I_{u}$ rather than all KPIs:

$$
T_{u}=\sum_{\substack{v=1 \\ v \neq u}}^{6} \gamma_{u v}
$$

Step 6. Calculate the normalized weight of any KPI.

$$
W K_{u}=\frac{T_{u}}{\sum_{u=1}^{6} T_{u}}
$$

Table 3. Matrix of KPI's score against DM's opinion

\begin{tabular}{lllllll}
\hline KPI & $\mathrm{DM}$ & & & & & \\
\cline { 2 - 7 } & $\mathrm{DM}_{1}$ & $\mathrm{DM}_{2}$ & $\ldots$ & $\mathrm{DM}_{\mathrm{j}}$ & $\ldots$ & $\mathrm{DM}_{\mathrm{m}}$ \\
\hline$K P I_{1}=C P I$ & $\mathrm{~W}_{11}$ & $\mathrm{~W}_{12}$ & $\ldots$ & $\mathrm{W}_{1 \mathrm{j}}$ & $\ldots$ & $\mathrm{W}_{1 \mathrm{~m}}$ \\
$K P I_{2}=S P I$ & $\mathrm{~W}_{21}$ & $\mathrm{~W}_{22}$ & $\ldots$ & $\mathrm{W}_{2 \mathrm{j}}$ & $\ldots$ & $\mathrm{W}_{2 \mathrm{~m}}$ \\
$K P I_{3}=Q P I$ & $\mathrm{~W}_{31}$ & $\mathrm{~W}_{32}$ & $\ldots$ & $\mathrm{W}_{3 \mathrm{j}}$ & $\ldots$ & $\mathrm{W}_{3 \mathrm{~m}}$ \\
$K P I_{4}=S S P I$ & $\mathrm{~W}_{41}$ & $\mathrm{~W}_{42}$ & $\ldots$ & $\mathrm{W}_{4 \mathrm{j}}$ & $\ldots$ & $\mathrm{W}_{4 \mathrm{~m}}$ \\
$K P I_{5}=S F P I$ & $\mathrm{~W}_{51}$ & $\mathrm{~W}_{52}$ & $\ldots$ & $\mathrm{W}_{5 \mathrm{j}}$ & $\ldots$ & $\mathrm{W}_{5 \mathrm{~m}}$ \\
$K P I_{6}=C R P I$ & $\mathrm{~W}_{61}$ & $\mathrm{~W}_{62}$ & $\ldots$ & $\mathrm{W}_{6 \mathrm{j}}$ & $\ldots$ & $\mathrm{W}_{6 \mathrm{~m}}$ \\
\hline
\end{tabular}

\section{3. $\otimes \operatorname{GIEAC}_{(t)}$ calculations}

New grey improved time estimate at completion $\left(\otimes G I E A C_{(t)}\right)$ is presented as follows:

$$
\begin{gathered}
P F=\otimes Z_{1} \times \otimes C P I+\otimes Z_{2} \times \otimes S P I_{(t)}+\otimes Z_{3} \times \otimes Q P I+\otimes Z_{4} \times \otimes S F P I+ \\
\otimes Z_{5} \times \otimes T R P I
\end{gathered}
$$

$\otimes \operatorname{GIEAC}_{(t)}=A D+$

$\frac{P D-\otimes E S}{\otimes Z_{1} \times \otimes C P I+\otimes Z_{2} \times \otimes S P I_{(t)}+\otimes Z_{3} \times \otimes Q P I+\otimes Z_{4} \times \otimes S F P I+\otimes Z_{5} \times \otimes T R P I}$

where $\otimes Z_{i}$ denotes the weighting of each KPI used in the formation of $\otimes$ $\operatorname{GIEAC}_{(t)}$, is obtained by W-TODIM method which is proposed in Section 3.2.

\subsection{Ranking}

A method proposed by Liu(2016) is applied for comparison of grey numbers whichaccording to this method, the steps of the grey ranking method are presented as follows:

(1) An ideal grey number $\otimes A^{*}$ is determined as follows: 
Amin Eshghi, Seyed M. Mousavi, Jurgita Antucheviciene, Vahid Mohagheghi

$\otimes A^{*}=\left[\max \left(\underline{A_{1}} \cdot \underline{A_{2}}\right) \cdot \max \left(\overline{A_{1}} \cdot \overline{A_{2}}\right)\right]=\left[\underline{A^{*}} \cdot \overline{A^{*}}\right]$

(2) The crisp output of $\otimes A_{1}$ and $\otimes A^{*}\left(\operatorname{Co}\left(\otimes A_{1} \cdot \otimes A^{*}\right)\right)$ and $\operatorname{Co}\left(\otimes A_{2} \cdot \otimes A^{*}\right)$ is computed as follows:

$\operatorname{Co}\left(\otimes A_{1} \cdot \otimes A^{*}\right)=\left(\overline{A_{1}}-\underline{A^{*}}\right)^{2} / 2$

$\operatorname{Co}\left(\otimes A_{2} \cdot \otimes A^{*}\right)=\left(\overline{A_{2}}-\underline{A^{*}}\right)^{2} / 2$

(3) Specify status of $\otimes A_{1}$ and $\otimes A_{2}$ relative to each other:

(i) If $\operatorname{Co}\left(\otimes A_{1} \cdot \otimes A^{*}\right)<\operatorname{Co}\left(\otimes A_{2} \cdot \otimes A^{*}\right)$, then $\otimes A_{1}<\otimes A_{2}$;

(ii) If $\operatorname{Co}\left(\otimes A_{1} \cdot \otimes A^{*}\right)=C o\left(\otimes A_{2} \cdot \otimes A^{*}\right)$, then $\otimes A_{1}=\otimes A_{2}$;

(iii) If $\operatorname{Co}\left(\otimes A_{1} \cdot \otimes A^{*}\right)>\operatorname{Co}\left(\otimes A_{2} \cdot \otimes A^{*}\right)$, then $\otimes A_{1}>\otimes A_{2}$.

\subsection{Interpretation}

Interpretation of the EV estimation's results is vital in order to arrive at a proper conclusion on the future status of the project in terms of cost and time. With regards to conventional procedure of $\mathrm{EV}$, estimated cost and time have to be comparedwith BAC and PD, respectively.In Tables 4 and 5, comparisons and related explanation are provided.

Table 4. Explanationof $\otimes G I E A C_{(\$)}{ }^{\prime} s$ scenarios

\begin{tabular}{cll}
\hline Scenario & Status & Explanation \\
\hline 1 & $\operatorname{Co}\left(\otimes G I E A C_{(\$)} \cdot \otimes A^{*}\right)=\operatorname{Co}\left(B A C . \otimes A^{*}\right)$ & $\begin{array}{l}\text { The project will be completed at a } \\
\text { similar cost to the BAC. }\end{array}$ \\
\hline 2 & $\operatorname{Co}\left(\otimes G I E A C_{(\$)} \otimes A^{*}\right)<\operatorname{Co}\left(B A C . \otimes A^{*}\right)$ & $\begin{array}{l}\text { The project will be completed at a } \\
\text { lower cost than the BAC. }\end{array}$ \\
\hline 3 & $\operatorname{Co}\left(\otimes G I E A C_{(\$)} \cdot \otimes A^{*}\right)>\operatorname{Co}\left(B A C \cdot \otimes A^{*}\right)$ & $\begin{array}{l}\text { The project will be completed with } \\
\text { more cost than the BAC. }\end{array}$ \\
\hline
\end{tabular}

Table 5. Explanation of $\otimes \operatorname{GIEAC}_{(t)}{ }^{\prime} \operatorname{sscenarios}$

\begin{tabular}{cll}
\hline Scenario & Status & Explanation \\
\hline 1 & $\operatorname{Co}\left(\otimes G I E A C_{(t)} \cdot \otimes A^{*}\right)=\operatorname{Co}\left(P D \cdot \otimes A^{*}\right)$ & $\begin{array}{l}\text { The project will be completed at a similar } \\
\text { time to the BAC. }\end{array}$ \\
\hline 2 & $\operatorname{Co}\left(\otimes G I E A C_{(t)} \cdot \otimes A^{*}\right)<\operatorname{Co}\left(P D \cdot \otimes A^{*}\right)$ & $\begin{array}{l}\text { The project will be completed at a lower } \\
\text { time than the PD. }\end{array}$ \\
\hline 3 & $\operatorname{Co}\left(\otimes G I E A C_{(t)} \cdot \otimes A^{*}\right)>\operatorname{Co}\left(P D \cdot \otimes A^{*}\right)$ & $\begin{array}{l}\text { The project will be completed with more } \\
\text { time than the PD. }\end{array}$ \\
\hline
\end{tabular}




\section{Earned Value Analysis Approach by Extending New Key Performance Indicators in a New Framework under a Grey Environment}

\section{Case study}

In this section, a case study from the recent literature (Salari et al., 2014)which refers to a construction project is presented and solved to illustratetheefficiency of the proposed grey model in earned value methodology. In this case, the first level ofwork breakdown structure includes six activities,such as roofing, interior, electrical, plumbing, framing, and concrete that each of them is divided into three work packages.For simplicity, we deal only with the WBS in first and second levels, despite the fact that it didnot limited to the two levels.

This case is take long a period of 14 months that the PV and AC of the project up to month 8 are brought in Table 6 . Also,linguistic terms of $\otimes G P P_{i}$ and BACfor each activity are depicted in Table 7.

Table 6. PV and AC of the project (Salari et al., 2014)

\begin{tabular}{cccccccc}
\hline Month & 1 & 2 & 3 & 4 & 5 & 6 & 7 \\
\hline PV & 4000 & 8000 & 11500 & 14000 & 16500 & 19600 & 23200 \\
AC & 3500 & 7500 & 10100 & 14460 & 16760 & 19360 & 22460 \\
\hline Month & 8 & 9 & 10 & 11 & 12 & 13 & 14 \\
\hline PV & 27500 & 28000 & 31500 & 37000 & 42500 & 47500 & 49500 \\
AC & 24410 & & & & & & \\
\hline
\end{tabular}

The linguistic terms of activities are transformed into $\otimes G P P_{i}$ using Table 1 and are stated in Table 7.

By regarding Eq. (2), for instance $\otimes E V_{12}$ is calculated as follows:

$\otimes E V_{12}=\otimes G P P_{12} * B A C_{12}=[0.0 .1] \times 1200=[0.120]$

Table 7. Information of activites (Salari et al., 2014) and their earned value

\begin{tabular}{llllll}
\hline & Activity & $\mathrm{BAC}(\$)$ & Progress & $\otimes G P P_{i}$ & $\otimes E V_{\mathrm{i}}$ \\
\hline 1 & Pour foundation & 8000 & Completed & {$[1,1]$} & {$[8000,8000]$} \\
2 & Install patio & 3400 & Very high & {$[0.9,1]$} & {$[3060,3400]$} \\
3 & Stairway & 2500 & half & {$[0.4,0.5]$} & {$[1000,1250]$} \\
4 & Frame exterior walls & 2500 & Not started & {$[0,0]$} & {$[0,0]$} \\
5 & Frame interior walls & 3000 & Low & {$[0.1,0.3]$} & {$[300,900]$} \\
6 & Install roofing trusses & 1250 & Not started & {$[0,0]$} & {$[0,0]$} \\
7 & Install water lines & 1900 & Low & {$[0.1,0.3]$} & {$[190,570]$} \\
8 & Install gas lines & 2300 & Low & {$[0.1,0.3]$} & {$[230,690]$} \\
9 & Install bath and fixture & 850 & Not started & {$[0,0]$} & {$[0,0]$} \\
10 & Install wiring & 950 & Very low & {$[0,0.1]$} & {$[0,95]$} \\
11 & Install outlet/switches & 1350 & Not started & {$[0,0]$} & {$[0,0]$} \\
12 & Install fixtures & 1200 & Very low & {$[0,0.1]$} & {$[0,120]$}
\end{tabular}


Amin Eshghi, Seyed M. Mousavi, Jurgita Antucheviciene, Vahid Mohagheghi

\begin{tabular}{llllll}
\hline 13 & Install drywalls & 2400 & half & {$[0.4,0.5]$} & {$[960,1200]$} \\
14 & Install carpets & 3200 & Not started & {$[0,0]$} & {$[0,0]$} \\
15 & Paintings & 5600 & Less than half & {$[0.3,0.4]$} & {$[1680,2240]$} \\
16 & Install felt & 3600 & more than half & {$[0.5,0.6]$} & {$[1800,2160]$} \\
17 & Install shingles & 2600 & Very low & {$[0,0.1]$} & {$[0,260]$} \\
18 & Install vents & 2900 & Not started & {$[0,0]$} & {$[0,0]$} \\
\hline
\end{tabular}

Based on Table 7 and Eq. (3), the overall EV for all the activities is as below:

$\otimes E V_{O}=\sum_{i=1}^{18} \otimes E V_{i}=[17220.20885]$

The PV and AC ineighth month are \$27,500 and \$24,410, respectively, as regards to Table 6. Hence, using Eq.(4) and Eq. (5),we can obtain theSPI and CPI,respectively.

$\otimes S P I=\left[\frac{17220}{27500} \cdot \frac{20885}{27500}\right]=[0.6261 \cdot 0.7594]$

$\otimes C P I=\left[\frac{17220}{24410} \cdot \frac{20885}{24410}\right]=[0.7054 .0 .8555]$

As stated in the second part of this study, the earned schedule calculations are computed in the following.The calculation of the ES is based on projecting each of the two members of grey $\otimes E V_{O}$ on the baseline as given inTable 7. For instance, $\mathrm{ES}_{\mathrm{i}}$ is obtained as follows:

$t_{1}=5$ as $P V_{5}<E_{1}^{U}=17720<P V_{6}$; therefore,

$E S_{1}=N_{1}+\left(\frac{E_{1}-P V_{5}}{P V_{6}-P V_{5}}\right)=5+\left(\frac{17720-16500}{19600-16500}\right)=5.2322$

$\otimes E S=[5.2322$ ، 6.3569]

The $\otimes S P I_{t}$ could be determined by Eq. (10):

$\otimes S P I_{t}=\frac{\otimes E S}{A D}=[5.2322 / 8 \cdot 6.3569 / 8]=[0.654 .0 .794]$

Then, the values of other KPIsare showed in Table8:

Table 8. Detailed calculation of KPIs

\begin{tabular}{lllll}
\hline KPI & Influential factors & IF weight & IF value & KPI amount \\
\hline \multirow{2}{*}{ QPI } & Rework/defects & {$[0.4091,0.3846]$} & {$[0.4444,0.5556]$} & \\
& Strategic quality & & & \\
& management & {$[0.1818,0.1923]$} & {$[0.5556,0.6667]$} & {$[0.5043,0.6931]$} \\
& Personnel quality training & {$[0.1818,0.1923]$} & {$[0.4444,0.5556]$} & \\
& Nonconformance rate & {$[0.2273,0.2308]$} & {$[0.6667,1]$} & \\
\cline { 2 - 5 } SSPI & Conflicts/disputes/claims & {$[0.6,0.5263]$} & {$[0.8333,1]$} & \\
& Change orders & {$[0.0667,0.1579]$} & {$[0.6667,0.8333]$} & {$[0.6409,0.9538]$} \\
& Stakeholder satisfaction & {$[0.3333,0.3158]$} & {$[0.5,0.6667]$} & \\
\cline { 2 - 5 }
\end{tabular}




\section{Earned Value Analysis Approach by Extending New Key Performance Indicators in a New Framework under a Grey Environment}

\begin{tabular}{|c|c|c|c|c|}
\hline \multirow{3}{*}{ SFPI } & Accident frequency ratio & {$[0.4,0.45]$} & {$[0.6667,1]$} & \multirow{3}{*}[0.4278,0.7241]{} \\
\hline & Safety equipment & {$[0.2667,0.25]$} & {$[0.1111,0.3333]$} & \\
\hline & Safety training & {$[0.3333,0.3$} & {$[0.4444,0.5556]$} & \\
\hline \multirow{5}{*}{ EPI } & Inflation & {$[0.3913,0.3571]$} & {$[0.5556,0.6667]$} & \multirow{5}{*}[0.5173,0.7013]{} \\
\hline & Energy & {$[0.1304,0.1429]$} & {$[0.4444,0.5556]$} & \\
\hline & Financial & {$[0.1739,0.1786]$} & {$[0.4444,0.5556]$} & \\
\hline & Cash flow & {$[0.1304,0.1429]$} & {$[0.6667,1]$} & \\
\hline & Developing cost & {$[0.1739,0.1786]$} & {$[0.5556,0.6667]$} & \\
\hline \multirow{3}{*}{ MPI } & Productivity & {$[0.2353,0.25]$} & {$[0.4444,0.5556]$} & \multirow{3}{*}[0.5686,0.835]{} \\
\hline & Errors & {$[0.5294,0.5]$} & {$[0.6667,1]$} & \\
\hline & Benefits to Costs & {$[0.2353,0.25]$} & {$[0.5556,0.6667]$} & \\
\hline \multirow{4}{*}{ PPI } & Environmental condition & {$[0.1364,0.1429]$} & {$[0,0.1111]$} & \multirow{4}{*}[0.469,0.7403]{} \\
\hline & Accidental (unscheduled) & {$[0.1818,0.1786]$} & {$[0.4444,0.5556]$} & \\
\hline & Government actions & {$[0.2727,0.3214]$} & {$[0.5556,0.6667]$} & \\
\hline & Sanctions & {$[0.4091,0.3571]$} & {$[0.6667,1]$} & \\
\hline \multirow{4}{*}{ CSPI } & Equipment & {$[0.3913,0.3448]$} & {$[0.4444,0.5556]$} & \multirow{4}{*}[0.4831,0.7623]{} \\
\hline & Consume resources & {$[0.1304,0.1379]$} & {$[0.6667,1]$} & \\
\hline & Capital Costs & {$[0.2174,0.2069]$} & {$[0.3333,0.4444]$} & \\
\hline & Manpower & {$[0.2609,0.3103]$} & {$[0.6667,1]$} & \\
\hline
\end{tabular}

On the basis ofsection 3.1, the $\otimes$ CRPIand $\otimes T R P I$ valuesare derived by summing thenormalized individual weight of a KPI multiplied with its value. It should be noted that the weights of KPIs in formation of the $\otimes T R P I$ formulation might be varied with the weights of KPIs in constitution of $\otimes C R P I$.

Table 9. Calculation of $\otimes C R P I$ with its elements

\begin{tabular}{lccc}
\hline KPI & Weight $\left(\otimes w_{i}\right)$ & KPI amount & CRPI amount \\
\hline EPI & {$[0.2899,0.2920]$} & {$[0.5172,0.7013]$} & \\
MPI & {$[0.1913,0.2108]$} & {$[0.5686,0.8349]$} & {$[0.4964,0.7691]$} \\
PPI & {$[0.2460,0.2545]$} & {$[0.4689,0.7402]$} & \\
CsPI & {$[0.2530,0.2620]$} & {$[0.4830,0.7622]$} & \\
\hline
\end{tabular}

Table 1. Calculation of TRPI with its elements

\begin{tabular}{lccc}
\hline KPI & Weight $\left(\otimes z_{k}\right)$ & KPI amount & TRPI amount \\
\hline EPI & {$[0.2765,0.2884]$} & {$[0.5172,0.7013]$} & \\
MPI & {$[0.2167,0.2553]$} & {$[0.5686,0.8349]$} & {$[0.488,0.791]$} \\
PPI & {$[0.1808,0.2120]$} & {$[0.4689,0.7402]$} & \\
CsPI & {$[0.2872,0.2827]$} & {$[0.4830,0.7622]$} & \\
\hline
\end{tabular}


Amin Eshghi, Seyed M. Mousavi, Jurgita Antucheviciene, Vahid Mohagheghi

Then,according to W-TODIM method,the weights of the CPI, SPI, QPI, SSPI, SFPI, and CRPI indicators are shown in Table 11 to influence on $\otimes G I E A C_{(\$)}$ and $\otimes \operatorname{GIEAC}_{(t)}$ formulae:

Table 2. Ultimate weights of KPIs in $\otimes G I E A C_{(\$)}$ and $\otimes G I E A C_{(\$)}$ formulae

\begin{tabular}{llll}
\hline$W K_{k p i}$ & W-TODIM Weights & $Z_{k p i}$ & W-TODIM Weights \\
\hline$W K_{1}$ & 0.197466 & $Z_{1}$ & 0.1572373 \\
$W K_{2}$ & 0.161020 & $Z_{2}$ & 0.1988687 \\
$W K_{3}$ & 0.177892 & $Z_{3}$ & 0.1890037 \\
$W K_{4}$ & 0.162255 & $Z_{4}$ & 0.1525147 \\
$W K_{5}$ & 0.152602 & $Z_{5}$ & 0.1479951 \\
$W K_{6}$ & 0.148763 & $Z_{6}$ & 0.1543801 \\
\hline
\end{tabular}

Now, computing the $\otimes G I E A C_{(\$)}$ and $\otimes G I E A C_{(\$)}$ are feasible as bellows:

$\otimes \operatorname{GIEAC}_{(\$)}=[60439.82 .80748 .51]$

$\otimes \operatorname{GIEAC}_{(t)}=[17.57351 \cdot 23.3106]$

\section{Discussion of results}

According to the responses obtained in the previous section, it is important that those responses be interprete the results. From Eq.(52) which $\otimes G I E A C_{(\$)}$ is in range of 60439,82 to 80748,51 and in according to the ranking methodCo( $(\otimes$ $\left.\operatorname{GIEAC}_{(\$)} \cdot \otimes A^{*}\right)=206221444.8$ is higher than $\operatorname{Co}\left(\mathrm{BAC} . \otimes \mathrm{A}^{*}\right)=59839830.82$. So, the project would be completed with more cost than the BAC. For time analysis, the range of $\otimes G I E A C_{(t)}$ is between 17,5735 and 23,3106. It is obtained that $\operatorname{Co}(\otimes$ $\left.\operatorname{GIEAC}_{(t)} \cdot \otimes A^{*}\right)$ is equal to16.4571 and $\operatorname{Co}\left(\mathrm{PD} . \otimes A^{*}\right)$ is equal to6.3849. Thus, for time analysis could be stated that the project would be completed with more time than the PD.

As stated earlier, one of the parameters that in turn affects $\otimes G I E A C_{(\$)}$ and $\otimes \operatorname{GIEAC}_{(t)}$ isgreyprogresspercents of each activities.In this regard, a sensitivity analysis is accomplishedto determine the effects of different grey progress percentsusing montecarlo simulation. Thus, the progress percent of any activity is modified and the main concentration is the estimations of results. A total of 8,000 iterations are run. The related simulation results are demonstrated in Table12, Figs.1 and 2. 


\section{Earned Value Analysis Approach by Extending New Key Performance Indicators in a New Framework under a Grey Environment}

Table 3. Summary of simulation results

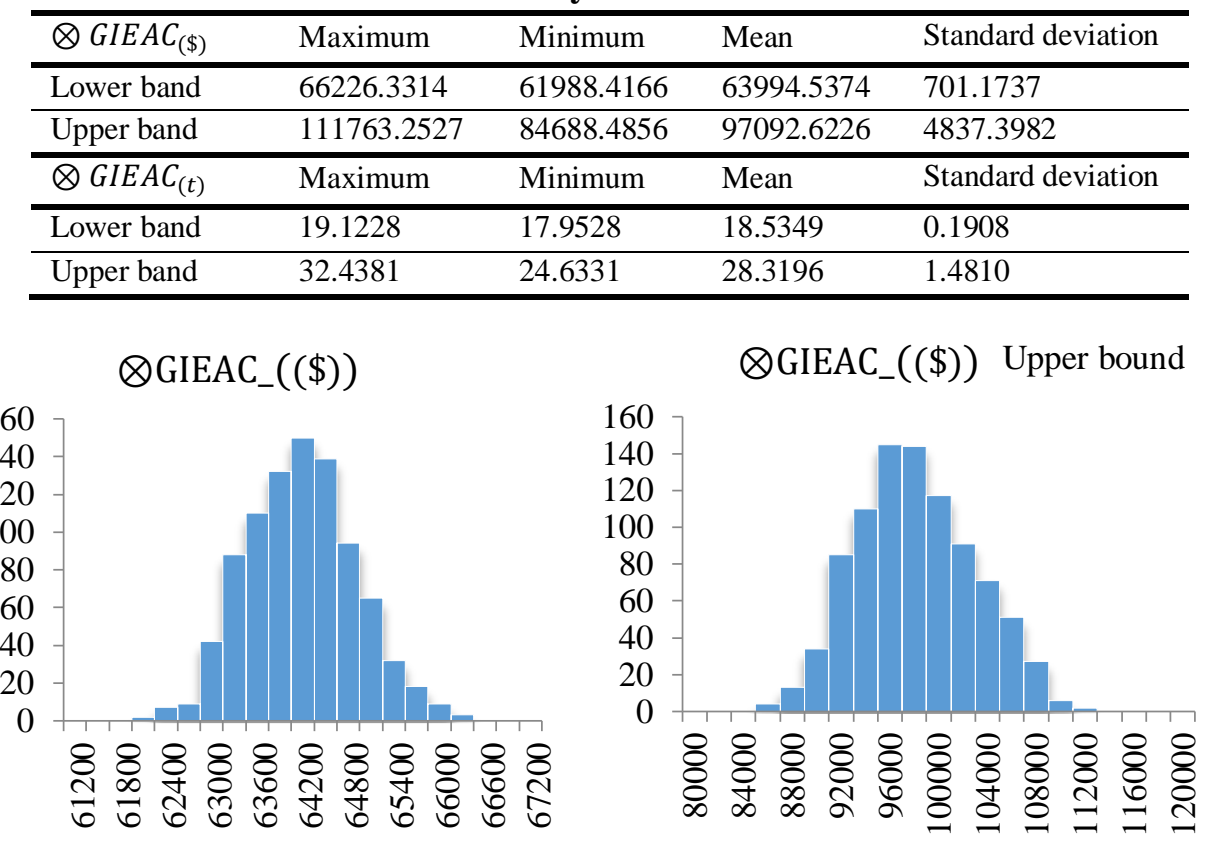

Figure 1. The $\otimes \operatorname{GIEAC}_{(\$)}$ simulation

Lower bound

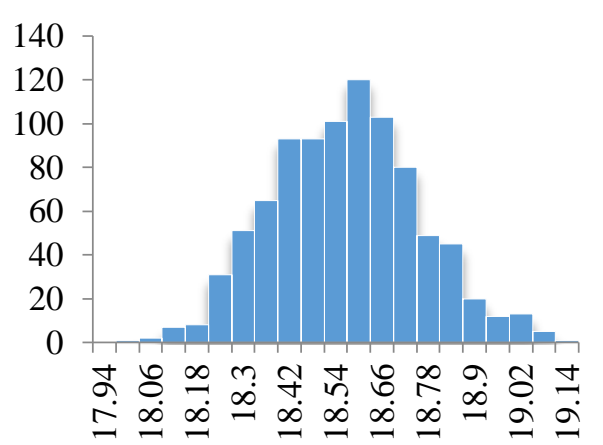

$\otimes \operatorname{GIEAC}_{-}((\mathrm{t}))$

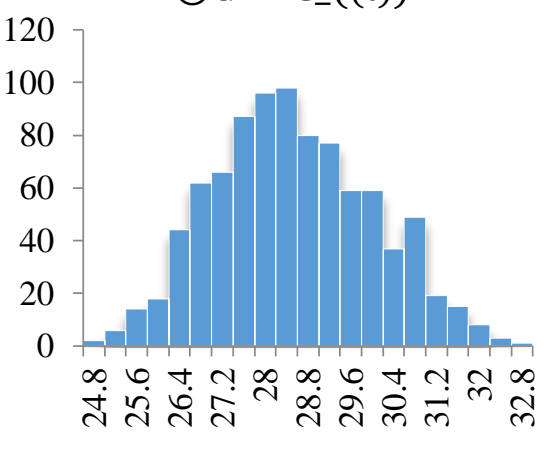

Figure 2.The $\otimes \operatorname{GIEAC}_{(t)}$ simulation

\section{Conclusion}

Nature of projects is gradually changing to more sophisticated and dynamic manner, particularly in construction projects. Several approaches that consider uncertainty in many ways have been provided significant for the construction 
Amin Eshghi, Seyed M. Mousavi, Jurgita Antucheviciene, Vahid Mohagheghi

project to improve their performance and secure project success. This paperapplied the grey theoryto handle the uncertainty for itsprecision and simplicity in modeling. In the first part, a new grey-based EV analysis was presented to show the flexibility in calculations.In second part, several KPIs had beenassessed to focus on various aspects of a construction project whereas other past versions were merely concentrated on four KPIs to formulating the cost and time estimations at completion. Moreover, newW-TODIM method was applied to assign the weights of six KPIs in estimations formulae in the grey environment. The practitioner can comfortably understand the status of project by successful implementation of the approach in a construction environment. The results show that the integration of key performance indicators leads to better analysis, as well as more accurate prediction and presentation of information. Hence, project managers can monitor the status condition of critical ingredient comprehensively and make informed conscious decisions by maintaining the simplicity of analyzes.To depicts the model's applicability, a case study for construction project from the literature was selected and solved precisely. In order to expand the study and as future research, fuzzy clustering methods of the factors influencing project risks can be used to determine the earned value.

\section{REFERENCES}

[1] Anantatmula, V. S. \& Fan, Y. (2018),Risk Management Strategies for Project Success. In Research, Practices, and Innovations in Global Risk and Contingency Management. IGI Global, 250-267;

[2] Bai, C. \&Sarkis, J. (2013),A Grey-based DEMATEL Model for Evaluating Business Process Management Critical Success Factors.International Journal of Production Economics 146(1), 281-292;

[3] Banihashemi, S., Hosseini, M. R., Golizadeh, H. \&Sankaran, S. (2017),Critical Success Factors (Csfs) for Integration of Sustainability into Construction Project Management Practices in Developing Countries. International Journal of Project Management 35(6), 11031119;

[4] Bhattacharyya, R. (2015),A Grey Theory Based Multiple Attribute Approach for R\&D Project Portfolio Selection. Fuzzy Information and Engineering 7(2), 211-225; 
Earned Value Analysis Approach by Extending New Key Performance Indicators in a New Framework under a Grey Environment

[5] Ekhtiari, M., Yadegari, E. \&Sadidi, G. (2016), Ranking

Entrepreneurship Main Risks in Non-Profit Financial Funds by TODIM Technique under Grey Condition (A Case Study in Iran).Economic Computation \& Economic Cybernetics Studies \& Research, 50(3), 319-336, ASE Publishing;

[6] Gomes, L. F. A. M. \& Lima, M. M. P. P. (1992),TODIM: Basics and Application to Multicriteria Ranking of Projects with Environmental Impacts. Foundations of Computing and Decision Sciences 16(4), 113127 ;

[7] Kerzner, H. (2017), Project Management Metrics, Kpis, and Dashboards: A Guide to Measuring and Monitoring Project Performance. John Wiley \& Sons;

[8] Lin, Y., Chen, M. Y. \& Liu, S. (2004),Theory of Grey Systems: Capturing Uncertainties of Grey Information. Kybernetes 33(2), 196-218;

[9] Liu, H. T. \& Cheng, H. S. (2016),An Improved Grey Quality Function Deployment Approach Using the Grey TRIZ Technique. Computers \& Industrial Engineering 92, 57-71;

[10] Noori, S., Bagherpour, M. \&Zareei, A. (2008),Applying Fuzzy Control Chart in Earned Value Analysis: A New Application. World Applied Sciences Journal 3(4), 684-690;

[11] Parmenter, D. (2015), Key Performance Indicators: Developing, Implementing, and Using Winning Kpis. John Wiley \& Sons;

[12] PMI (2013), A Guide to the Project Management Body of Knowledge (PMBOK Guide). Fifth edition. Project Management Institute;

[13] Rahimnia, F., Moghadasian, M., \&Mashreghi, E. (2011),Application of Grey Theory Approach to Evaluation of Organizational Vision.Grey Systems: Theory and Application 1(1), 33-46;

[14] RazaviHajiagha, S. H., Akrami, H., Hashemi, S. S. \&Mahdiraji, H. A. (2015),An Integer Grey Goal Programming for Project Time, Cost and Quality Trade-Off. Engineering Economics 26(1), 93-100;

[15] Rostami, A, Sommerville, J, Wong, I.L, Lee, C. (2015), Risk Management Implementation in Small and Medium Enterprises in the UK Construction Industry. Engineering, Construction and Architectural Management 22(1): 91-107; 
Amin Eshghi, Seyed M. Mousavi, Jurgita Antucheviciene, Vahid Mohagheghi

[16] Salari, M., Bagherpour, M. \& Wang, J. (2014), A Novel Earned Value Management Model Using Z-Number.International Journal of Applied Decision Sciences 7(1), 97-119;

[17] Simion, C. P. \& Marin, I. (2018),Project Cost Estimate at Completion: Earned Value Management versus Earned Schedule-based Regression Models. A Comparative Analysis of the Models Application in the Construction Projects in Romania.Economic Computation \& Economic Cybernetics Studies \& Research 52(3), 215-216, ASE Publishing;

[18] Slavek, N. \&Jović, A. (2012), Application of Grey System Theory to Software Projects Ranking. Automatika: časopiszaautomatiku, mjerenje, elektroniku, računarstvoikomunikacije, 53(3), 284-293;

[19] Taillandier, F, Taillandier, P, Tepeli, E, Breysse, D, Mehdizadeh, R, Khartabil, F. (2015), A Multi-agent Model to Manage Risks in Construction Project (SMACC).Automation in Construction, 58, 1-18; 